\title{
Oxygen vacancy-induced topological nanodomains in ultrathin ferroelectric films
}

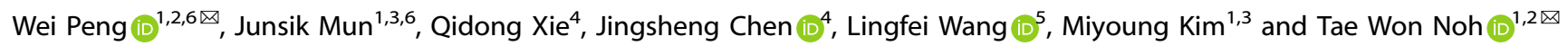

Oxygen vacancy in oxide ferroelectrics can be strongly coupled to the polar order via local strain and electric fields, thus holding the capability of producing and stabilizing exotic polarization patterns. However, despite intense theoretical studies, an explicit microscopic picture to correlate the polarization pattern and the distribution of oxygen vacancies remains absent in experiments. Here we show that in a high-quality, uniaxial ferroelectric system, i.e., compressively strained $\mathrm{BaTiO}_{3}$ ultrathin films (below $10 \mathrm{~nm}$ ), nanoscale polarization structures can be created by intentionally introducing oxygen vacancies in the film while maintaining structure integrity (namely no extended lattice defects). Using scanning transmission electron microscopy, we reveal that the nanodomain is composed of swirling electric dipoles in the vicinity of clustered oxygen vacancies. This finding opens a new path toward the creation and understanding of the long-sought topological polar objects such as vortices and skyrmions.

npj Quantum Materials (2021)6:48; https://doi.org/10.1038/s41535-021-00349-y

\section{INTRODUCTION}

In the past 10 years, an intriguing query in ferroelectricity studies has been the existence of topological arrangement of electric dipoles such as vortices and skyrmions in magnetic systems ${ }^{1-10}$. These topological objects hold considerable interests from both fundamental and practical aspects. However, unlike spins that are less coupled to the lattice strain and can rotate away from the high-symmetry direction to favor noncollinear, chiral arrangements, the arrangement of electric dipoles is strongly restricted by the crystal symmetry ${ }^{9}$. For example, ferroelectric systems usually exhibit Ising-type domain walls, of which the electric dipoles gradually vanish and then reverse along the polar axis, contrasting the chiral Bloch- or Neel-type domain walls in magnetic systems ${ }^{11}$. This difference renders the pursuit of ferroelectric topological objects particularly challenging. To date, successful cases have been only reported in a limited number of ferroelectric film systems, including $\mathrm{PbTiO}_{3}$ and $\mathrm{BiFeO}_{3}$ films, where polar vortices $^{2,4,5}$, skyrmions ${ }^{3,10}$, and merons ${ }^{12}$ have been discovered. These were mainly achieved via designing mechanical and electrical boundary conditions to flatten the energy landscape of ground-state polar structures and bring about the rotation of polar vectors. Another rarely investigated pathway lies in the exploitation of ionic point defects, which can break the lattice symmetry locally via electric and strain fields. For example, hedgehog- and vortex-like polarization states were recently discovered in the vicinity of charged point defects in $\mathrm{BiFeO}_{3}$ thin films ${ }^{13}$. The charged defects were immobile cation impurities intentionally introduced during film growth. Despite some promise, this success is limited to specific materials with complex growth design, thus falling short of generality.

Oxygen vacancies, as a prevalent type of point defects in oxide materials, have been revealed to play an influential role in the properties of oxide ferroelectrics on various aspects ${ }^{14,15}$. For example, it has been well documented that oxygen vacancies can be attracted and accumulated at the domain walls, heterointerfaces, and other lattice defects as pinning sites to cause unwanted polarization fatigue $\mathrm{e}^{16,17}$ but also trigger intriguing emergent phenomena in some cases ${ }^{18-20}$. A fundamental aspect to comprehend the role of oxygen vacancies is the microscopic picture of the interplay between oxygen vacancies and the polar order ${ }^{21-25}$. Theoretical studies using first-principles calculations and force fieldbased methods have managed to depict atomic pictures, where oxygen vacancies can cluster and significantly impact local polarization profiles via lattice distortion, e.g., inducing $180^{\circ}$ charged domain walls and forcing polarization to tilt away from the highsymmetry axis ${ }^{22-24}$. These findings imply that oxygen vacancies can be coupled to exotic polarization patterns with lower symmetries. However, the majority of experimental studies have so far only managed to identify the passive role of oxygen vacancies in their interaction with electric dipoles, e.g., electrostatic passivation on charged domain walls ${ }^{19,25,26}$. The more active role of oxygen vacancies in the creation of polarization domains has not been identified.

Here we show that high-quality, oxygen-deficient $\mathrm{BaTiO}_{3}$ films (with a thickness below $10 \mathrm{~nm}$ ), which are compressively strained on $\mathrm{SrTiO}_{3}$ (001) substrates, can exhibit an exotic, dynamic evolution from a uniform polarization state to exotic nanoscale polarization structures in an aging process under ambient conditions. By piezoelectric force microscopy (PFM) and spectroscopic techniques, we point out the critical role of oxygen vacancy clusters in such a dynamic process. Scanning transmission electron microscopy (STEM) further reveals that oxygen vacancies clusters give rise to polarization vortices without any extended lattice defects. This is in sharp contrast to the common observation in a uniaxial ferroelectric system, e.g., compressively strained $\mathrm{BaTiO}_{3}$ and $\mathrm{PbTiO}_{3}$ films with ideal chemistry, of which the tetragonal $c$ axis is strongly favored along the out-of-plane direction, thus exhibiting a pure out-of-plane polarization state ${ }^{27,28}$. Therefore, our finding will inspire future efforts to investigate and exploit the role of oxygen vacancies in the creation of exotic domain structures in ferroelectric materials.

\footnotetext{
${ }^{1}$ Center for Correlated Electron Systems, Institute for Basic Science, Seoul, Republic of Korea. ${ }^{2}$ Department of Physics and Astronomy, Seoul National University, Seoul, Republic of Korea. ${ }^{3}$ Department of Materials Science and Engineering and Research Institute of Advanced Materials, Seoul National University, Seoul, Republic of Korea. ${ }^{4}$ Department of Materials Science and Engineering, National University of Singapore, Singapore, Singapore. ${ }^{5} \mathrm{Hefei}$ National Laboratory for Physical Sciences at Microscale, University of Science and Technology of China, Hefei, China. ${ }^{6}$ These authors contributed equally: Wei Peng, Junsik Mun. ${ }^{凶}$ email: wei.peng@snu.ac.kr; twnoh@snu.ac.kr
} 


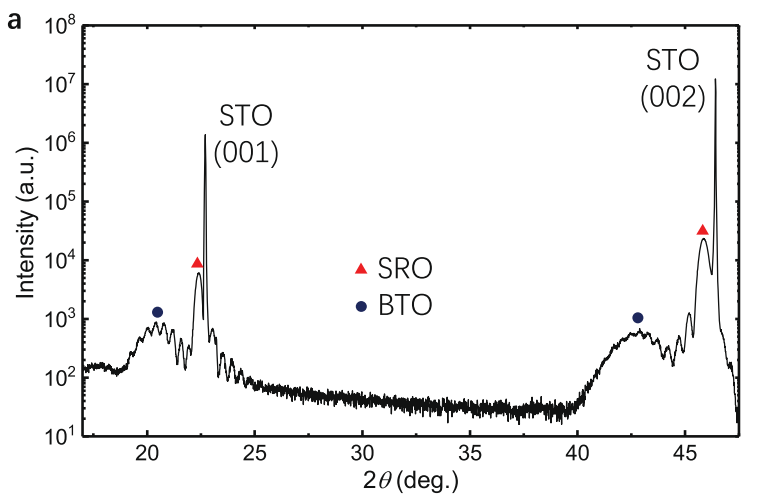

b

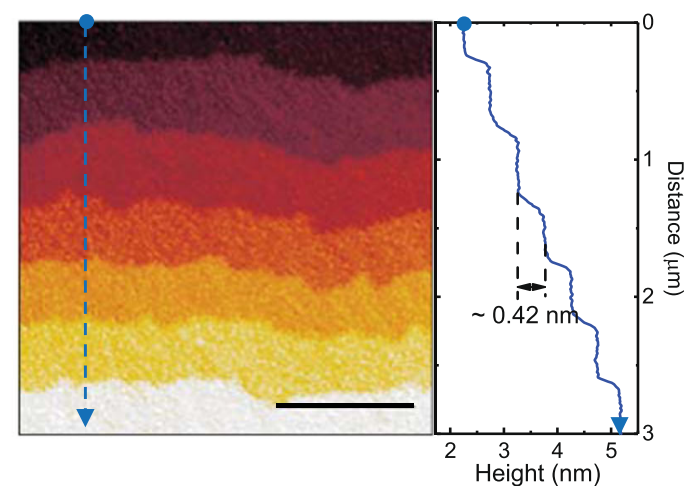

C

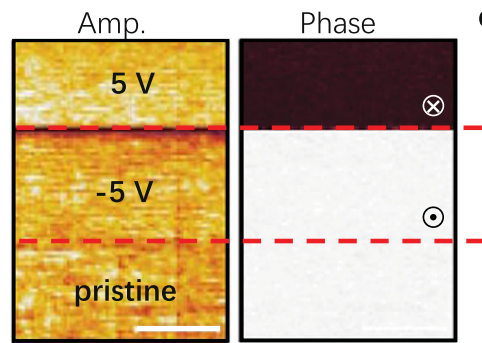

20 mTorr d

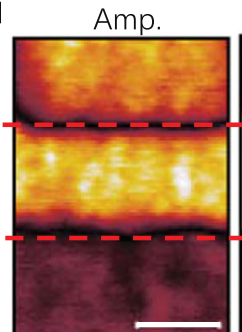

5 mTorr

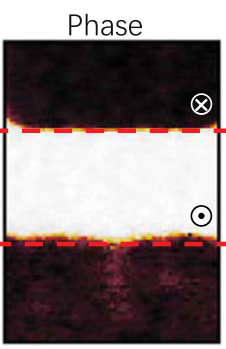

-

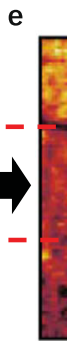

Amp.

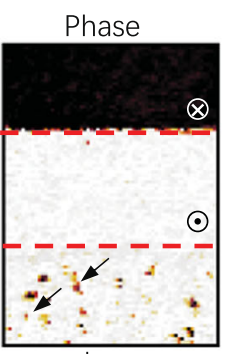

5 mTorr aged

Fig. 1 BTO film quality characterization and self-polarization examination. a $\theta-2 \theta$ scan of X-ray diffraction (XRD) and $\mathbf{b}$ atomic force microscopy (AFM) morphology of a 5-BTO film. The inset of (b) shows the height profile along the marked line in the AFM image. The scale bar for the AFM image is $1 \mu \mathrm{m}$. Piezoelectric force microscopy (PFM) images of a 20-BTO film (c) and a fresh 5-BTO film (d), with positively (field pointing from the film surface to the bottom electrode) and negatively biased, as well as pristine regions. $\mathbf{e}$ The aged domain structure of the 5-BTO film in air, with arrows indicating the locations of some nanodomains. The scale bar for all the PFM images is $1 \mu \mathrm{m}$.

\section{RESULTS}

\section{Oxygen pressure-dependent polarization structures by PFM}

$\mathrm{BaTiO}_{3}$ (BTO) films with a bottom $\mathrm{SrRuO}_{3}$ (SRO) electrode layer were grown on $\mathrm{TiO}_{2}$-terminated $\mathrm{SrTiO}_{3}(001)$ substrates by pulsed laser deposition $(P L D)^{29}$. The oxygen partial pressure for BTO growth was varied from 20 to 5 mTorr with the purpose of modulating the density of oxygen vacancies in the film. The high quality of BTO films grown under 5 mTorr (henceforth 5-BTO) was inferred from the diffraction fringes of X-ray diffraction (XRD) and the step-terrace morphology with unit-cell step height by atomic force microscopy (AFM, Fig. 1a, b). By XRD and AFM, we also confirmed the consistent quality of BTO films grown under higher oxygen pressures up to 20 mTorr (Supplementary Figs. 1 and 2).

We first examined the polarization state of the BTO films by PFM. As shown in Fig. 1C, the pristine BTO film grown under 20 mTorr (henceforth 20-BTO) exhibits the same phase contrast as the negatively biased region (where the electric field pointed from the bottom electrode to the top surface of the BTO film), suggesting a pure upward self-polarization (pointing from the bottom SRO electrode toward the top surface). This result is consistent with previous studies on oxygen-rich, ultrathin BTO films ${ }^{28,30}$. Such a preferred polarization orientation was believed to originate from a built-in field generated by surface anionic adsorbates such as hydroxyl (see Supplementary Discussion) ${ }^{27,31}$. By contrast, fresh BTO films grown in lower oxygen pressures, namely 5-BTO, exhibit a uniformly opposite self-polarization in average, namely pointing from the top surface down to the bottom electrode (Fig. 1d). More interestingly, the uniform downward polarization state is metastable and will gradually evolve to a stable "raisin-in-cake" polarization structure after aging in the air or vacuum for over one day (Fig. 1e). As shown in Fig. 2a, the aged polarization structure is composed of nanoregions with dark PFM phase contract (owning a lateral dimension of 100 to below $10 \mathrm{~nm}$ ), embedded in an upward polarization matrix. Figure $2 b$ shows the cross-section line profiles of the PFM phase and amplitude of two representative regions containing the nanostructures. As the nanostructures exhibit an opposite PFM phase compared to that of the upward polarization matrix, it seems to suggest that these nanostructures possess a downward polarization. However, we also note that the PFM amplitude of these nanostructures is significantly weaker than that of the upward polarization matrix. As PFM estimates an average polarization in the depth direction with a relatively large lateral dimension (tens of nanometers), this weaker amplitude may suggest an inhomogeneous polarization state in the nanostructures (which we will discuss in detail in the STEM results later) ${ }^{29}$. But for convenience, we will use "up domain" and "down domain" to refer to these two polarization structures by PFM in the following discussion, respectively.

The non-uniform polarization structure of the 5-BTO film should point to inhomogeneous distribution of local built-in fields $\left(E_{\text {in }}\right)$, which can be revealed by local PFM hysteresis loops (Fig. 2c). The PFM hysteresis loops were measured at different regions, from which we extracted their corresponding coercive fields $\left(E_{\mathrm{c}}\right)$ and $E_{\mathrm{in}}$ (Fig. 2c). As expected, the "up domain" typically exhibits an asymmetric hysteresis loop shifting to the positive field side, suggesting an $E_{\text {in }}$ pointing to the top surface. The corresponding $E_{\text {in }}$ can be then estimated from the difference of the positive coercive field $\left(E_{\mathrm{c}}{ }^{+}\right)$and the negative coercive field $\left(E_{\mathrm{c}}{ }^{-}\right)$, i.e., $E_{\text {in }}=\left(\left|E_{c}^{+}\right|-\left|E_{c}^{-}\right|\right) / 2$. Figure 2 d presents a statistic of $E_{\mathrm{c}}{ }^{+}, E_{\mathrm{c}}{ }^{-}$, and $E_{\text {in }}$ of the up domains at 30 different locations, showing an average $E_{\text {in }}$ of about $0.3 \mathrm{~V}$. The uniform up-polarization state of the 20-BTO film exhibits similarly asymmetric hysteresis loops with comparable $E_{\text {in }}$ (not shown here). Therefore, we believe that the upward polarizations in the two films share the same origin, namely surface anionic adsorbates. In comparison, the hysteresis loop of the "down domain" in the 5-BTO film is oppositely shifted with an average $E_{\text {in }}$ of about $-0.3 \mathrm{~V}$ (Fig. $2 \mathrm{~d}$ ). These results clearly suggest an additional factor in the "down domain," which produces an opposite $E_{\text {in }}$ to cancel the field from surface anionic adsorbates. 
a

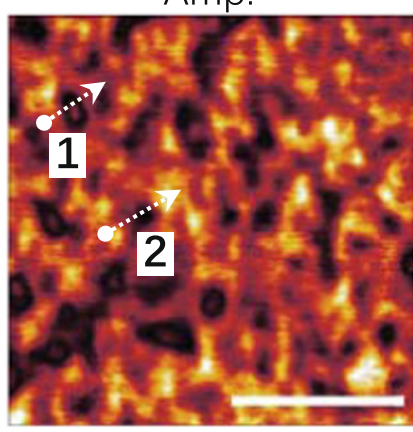

Phase

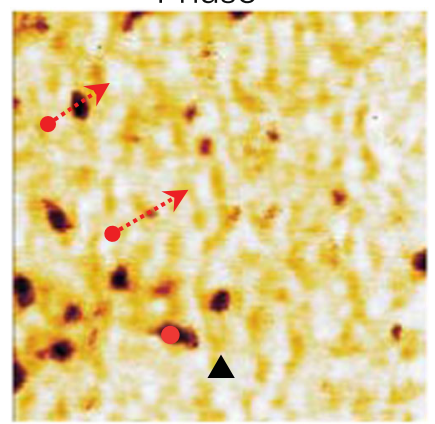

b

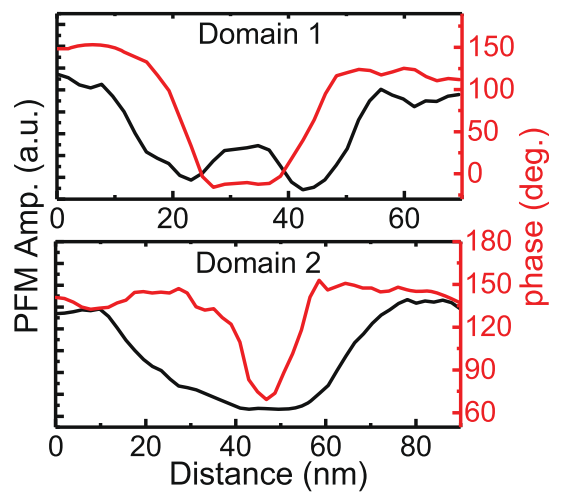

C

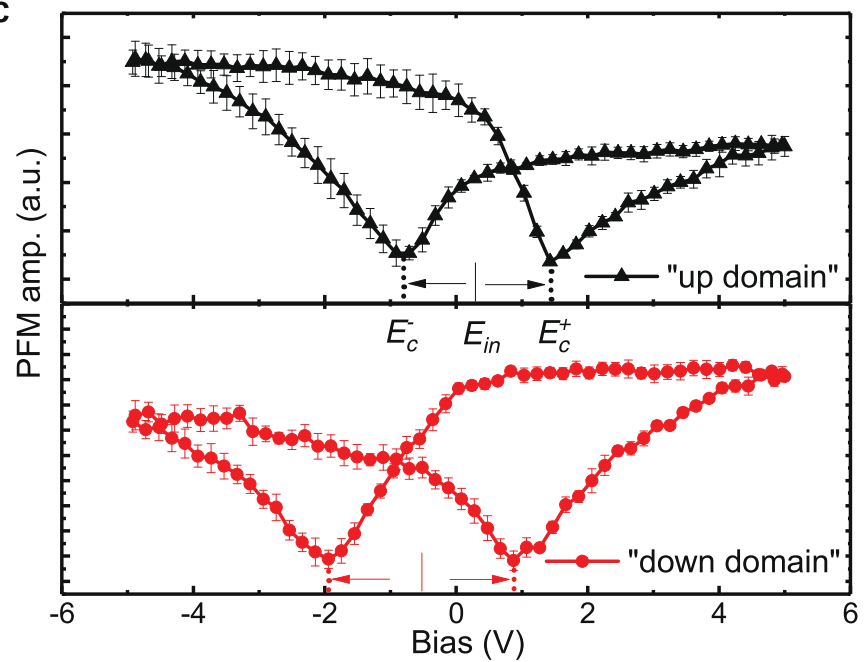

d

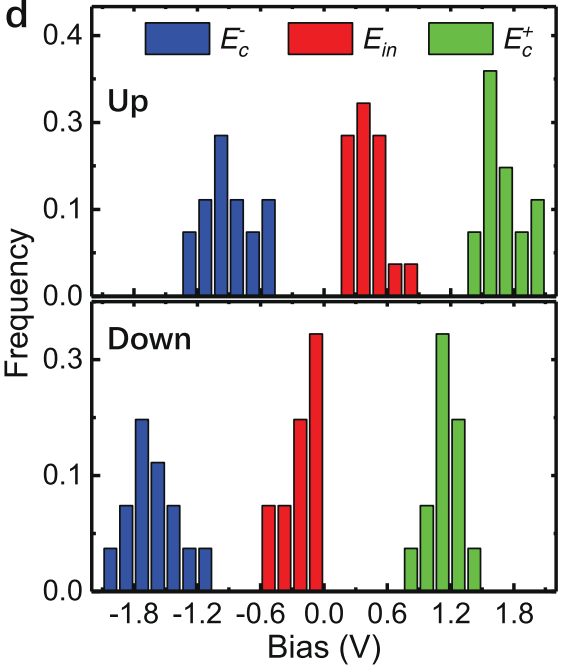

Fig. 2 Analysis of the nanodomains in 5-BTO by PFM. a Zoomed-in piezoelectric force microscopy (PFM) image of the nanodomains in the aged 5-BTO film with a scale bar of $200 \mathrm{~nm}$. b Cross-section line profiles of the phase and amplitude of the down domains indicated by arrows in (a). From the amplitude profile, domain wall can be distinguished from the domain interior for Domain 1 ( 20 nm), but impossible for Domain 2 with a smaller size $(<10 \mathrm{~nm})$. c Representative PFM hysteresis loops of up and down domains. While the up domain exhibits a loop shifting to the positive field side, the loop of the down domain shifts to the negative side. The hysteresis loops were an average of ten loops acquired from the same location, with the error bar representing a standard deviation. $\mathbf{d}$ Statistic of the extracted $E_{c}{ }^{+}, E_{c}{ }^{-}$, and $E_{i n}$ for 30 different up and down domains, respectively.

A variety of factors besides surface-adsorbed ions have been accounted for the self-polarization of epitaxial oxide ferroelectrics in previous studies. These include strain gradients ${ }^{32-34}$, interfacial electrostatic potential caused by valence discontinuity ${ }^{35}$ or Schottky junction at the electrode/ferroelectric interface ${ }^{36}$, and ionic point defects ${ }^{31,37}$. First of all, since our ultrathin BTO films are fully strained (Fig. 3b), strain gradients resulted from misfit strain relaxation can be ruled out as a possible factor in our study. Second, both BTO and SRO along the [001] direction have alternating stack of charge-neutral $\mathrm{AO}$ and $\mathrm{BO}_{2}$ layers $(\mathrm{A}$ is referred to the $A$ site cation and $B$ for $B$ site cation), thus proscribing the existence of interfacial electrostatic potential due to valence discontinuity. In addition, a Schottky junction usually generates an electrostatic potential uniformly in the film plane, thus favoring a homogeneous polarization state. Therefore, it is unlikely that interfacial electrostatic potentials play a vital role in the observed nanodomain structure of the 5-BTO film. Based on these considerations, we are inclined to ascribe the nanodomain structure to ionic point defects, or more specifically, oxygen vacancies. This supposition is consistent with the distinctive domain structures of the two films by just varying the oxygen pressure from 20 to $5 \mathrm{mTorr}$. A more detailed examination reveals that the nanodomains grow in size and density with the decrease of $\mathrm{O}_{2}$ partial pressure from 20 to 5 mTorr (Supplementary Fig. 3).
To further testify the critical role of oxygen vacancies, we post-annealed the 5 -BTO film at $500^{\circ} \mathrm{C}$ in $\mathrm{O}_{2}$ to remove possible oxygen vacancies. As expected, the non-uniform polarization structure disappeared and a pure upward self-polarization was created. Similarly, by post-annealing the 20-BTO film in ultrahigh vacuum, an analogous nanoscale polarization structure can be also built (Supplementary Fig. 4). These results strongly imply the critical role of oxygen vacancies in the current study.

Then an interesting question is how oxygen vacancies can bring about the dynamic evolution of the domain structure in the 5-BTO film. In the beginning, oxygen vacancies generated during film deposition tend to distribute uniformly in the film at the growth temperature $\left(700^{\circ} \mathrm{C}\right)$ as a result of high entropy ${ }^{38}$. Meanwhile, depending on the formation energy of oxygen vacancies in BTO and the oxidizability of the environment in the growth chamber, which may lead to two different cases. (1) Oxygen may escape from the top surface of BTO if the formation energy of oxygen vacancy is too small (that is difficult to be reoxidized). (2) Or considering a slightly oxidizing environment and the diffusivity of oxygen vacancies at such a high temperature, oxygen vacancies may be further attracted to the top surface by their affinity to oxygen on the free surface. In both cases, a density gradient of oxygen vacancies should form along the film thickness direction. Then after a relatively short cooling 

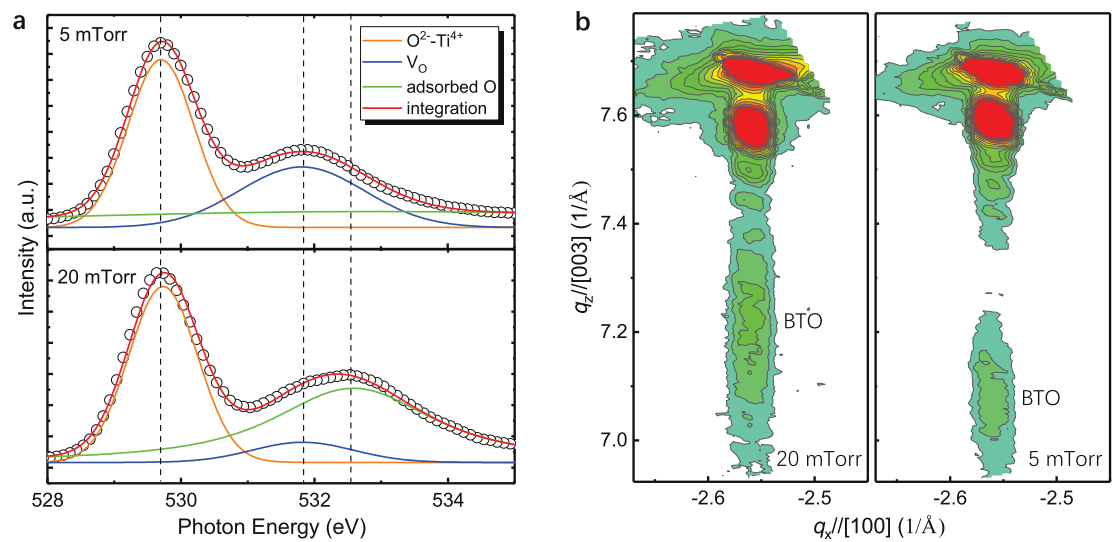

Fig. 3 Examination of oxygen vacancy population. a X-ray photoemission spectrum (XPS) of the O $1 \mathrm{~s}$ core level of BTO grown under 5 and 20 mTorr, respectively. The orange (centered $\sim 529.7 \mathrm{eV}$ ), green (centered $\sim 531.8 \mathrm{eV}$ ), and blue (centered $\sim 532.5 \mathrm{eV}$ ) fitted curves correspond to the $\mathrm{O}$ contributed by $\mathrm{O}^{2-}$ bounded with $\mathrm{Ti}^{4+}$ in $\mathrm{BTO}$, oxygen vacancies $\left(\mathrm{V}_{\mathrm{O}}\right)$, and surface-adsorbed $\mathrm{OH}^{-}$or $\mathrm{H}_{2} \mathrm{O}$, respectively. b Asymmetric reciprocal space mapping (RSM) of 5- and 20-BTO films near the STO (103) reciprocal lattice point. While both BTO films are fully strained (inferred from the commensurate in-plane lattice constants as that of the STO substrate), the out-of-plane lattice constants are extracted as 0.424 and $0.416 \mathrm{~nm}$ for 5- and 20-BTO films, respectively.

procedure $\left(\sim 1 \mathrm{~h}\right.$ with a cooling rate of $\left.10^{\circ} \mathrm{C} \min ^{-1}\right)$, the distribution of oxygen vacancies in the as-prepared 5-BTO film should remain in such a metastable state: namely uniform in the film plane but more populated at the top surface along the thickness direction. The population of oxygen vacancies with positive charges at the top surface of the film can then generate a built-in electric field, pointing downward to the substrate. This built-in field can well explain the as-observed uniform downward polarization in the fresh 5-BTO film ${ }^{31}$. Furthermore, oxygen vacancies have been well known for their aging and clustering behavior at room temperature as a result of large energy gain during clustering ${ }^{24,38,39}$. This aging and clustering process usually occurs with a relatively long relaxation time due to the low diffusion coefficient of oxygen vacancies at room temperature. Consequently, oxygen vacancies gradually evolve into inhomogeneously distributed clusters accompany with locally varying fields, which is consistent with the gradually emerged inhomogeneous polarization configurations observed by PFM.

\section{Examination of oxygen vacancy population}

To verify the existence of oxygen vacancy, we measured the photoemission spectra of $\mathrm{O} 1 \mathrm{~s}$ core levels by X-ray photoemission spectroscopy (XPS). As displayed in Fig. 3a, three peaks can be observed: namely one main peak centering at $\sim 529.7 \mathrm{eV}$, one satellite peak at $\sim 531.8 \mathrm{eV}$, and another at $\sim 532.5 \mathrm{eV}$. According to the literature, these three peaks have been assigned to the lattice bonded $\mathrm{O}$ in BTO, oxygen vacancies, and surface-adsorbed $\mathrm{OH}^{-}$ and $\mathrm{H}_{2} \mathrm{O}$, respectively ${ }^{40,41}$. As expected, besides the main peak of lattice bonded O, the 5-BTO sample shows a satellite peak mainly contributed by oxygen vacancies. In comparison, the 20-BTO sample exhibits a satellite shoulder with a major component of surface-adsorbed oxygen and a minor component of oxygen vacancies. This result is consistent with the scenario of surface anionic adsorbates-induced upward self-polarization in the 20BTO sample, and confirms populated oxygen vacancies in the 5BTO sample. In addition, we extracted a $c$-axis lattice constant of $\sim 0.424$ and $\sim 0.416 \mathrm{~nm}$ for the 5-BTO and 20-BTO films, respectively, from the reciprocal space mapping of their (103) planes (Fig. 3b). Such a significant elongation of the out-of-plane axis in the 5-BTO film in comparison to the 20-BTO film can also be ascribed to the well-known chemical expansion effect due to oxygen vacancies ${ }^{15}$.

\section{Atomic-scale observation of polarization structures by STEM}

Rich local fields including electrostatic forces and distorted lattice strains are usually accompany with oxygen vacancies ${ }^{15}$, we were thus intrigued to investigate the microscopic configuration of the electric dipoles in the vicinity of clustered oxygen vacancies in the ultrathin 5-BTO film by STEM. A large-scale low-angle annular dark-field (LAADF)-STEM image is shown in Fig. 4a, which displays cloudy contrast in BTO and suggests the existence of rich point defects, such as oxygen vacancies ${ }^{39}$. By further adjusting the contrast (Fig. 4b), the processed image shows cloudy regions of $\sim 10 \mathrm{~nm}$ or above in the BTO film. The contrast variation is consistent with the picture of oxygen vacancy clusters. Figure $4 \mathrm{c}$ displays a high-angle annular dark-field (HAADF)-STEM image of the 5-BTO/SRO/STO (001) heterostructure. The heterostructure shows sharp heterointerfaces with no extended defects throughout the films, demonstrating its high quality. The displacement vectors of the $\mathrm{Ti}$ cations with respect to the Ba cage in BTO are mapped in Fig. 4d with overlaid arrows. We note here that, due to rich oxygen vacancies, it is challenging to resolve oxygen positions via angular bright-field STEM, thus unable to acquire the local polarization vector accurately. Nevertheless, as the polarization of BTO is contributed by a dominant Ti displacement and a much smaller, opposite $\mathrm{O}$ displacement (relative to $\mathrm{Ba}$ ), the geometry of Ti displacement vectors should resemble the polarization patterns to a large extent (see Supplementary Discussion) ${ }^{42}$. Contrary to the well-established picture of $180^{\circ}$ domains with Ising-type domain walls in ultrathin films of uniaxial ferroelectrics, the electric dipoles in the 5-BTO film form complex rotation patterns (marked with the blue squares). Figure $4 \mathrm{e}$ shows the magnified vortex (Domain III) or half vortex (Domain I and II) ${ }^{5,13}$. It is noteworthy that these polarization patterns are prevailing throughout the sample in our STEM observation (Supplementary Fig. 6). To validate our STEM observation, we plot the distribution of the $\mathrm{Ti}$ displacement of both BTO and STO (Supplementary Fig. 5). As expected for a non-polar material, STO exhibits nearly vanishing $\mathrm{Ti}$ displacement. This result certifies the quality of our STEM image. By contrast, the Ti displacement of BTO has a rather dispersed distribution in both magnitudes (ranging from nearly 0 up to $30 \mathrm{pm}$ ) and directions, in consistence with the vortex-like polarization patterns.

\section{Origin of polarization nanodomains}

To reveal the origin of such exotic polarization pattern, we analyze the local strain state by lattice constant mapping and 

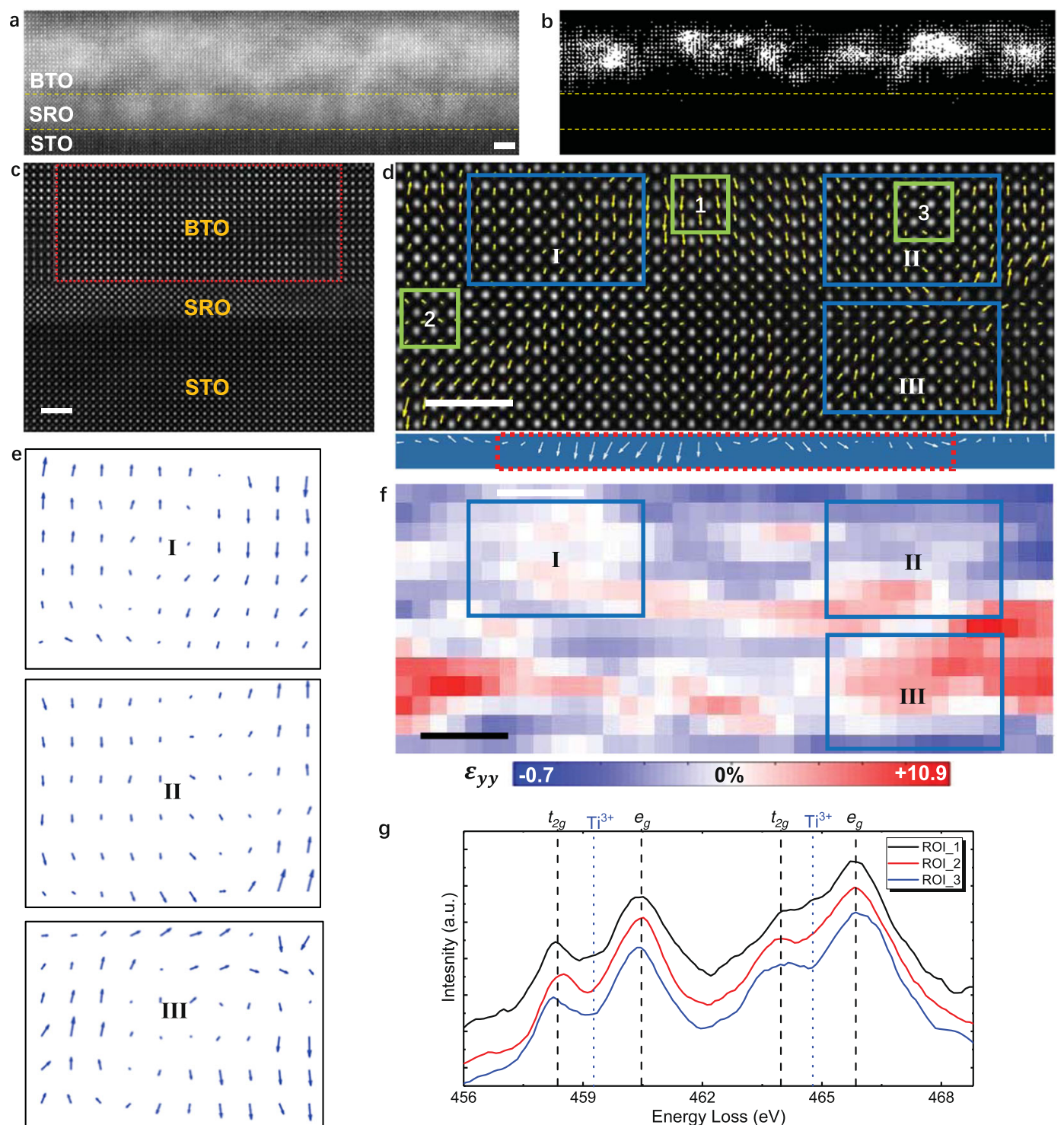

Fig. 4 STEM observation of 5-BTO. a A LAADF-STEM image of the 5-BTO/SRO/STO heterostructure. The cloudy contrast in BTO indicates the existence of extra strain fields due to oxygen vacancies. $\mathbf{b}$ The processed image of (a) with adjusted contrast to show clearly clustered regions with cloudy contrast in the middle of BTO. c A HAADF-STEM image of the 5-BTO/SRO/STO heterostructure, showing sharp heterointerfaces. d Ti-displacement vector mapping of BTO for the region indicated by the red square in (c). The lower panel shows the average Tidisplacement of each atomic column, with the red dashed square marking a dominant downward polarization state. e Magnified regions of Tidisplacement vectors for a half vortex (Domain I), half antivortex (Domain II), and vortex (Domain III), as indicated by the blue squares in (d). $\mathbf{f}$ Out-of-plane strain mapping of the same BTO region in (d), showing considerable inhomogeneity. $\mathbf{g}$ Ti- $L_{2,3}$ electron energy loss spectra (EELS) of region-of-interest (ROI)-1, 2, and 3, as marked by the green squares in (d). The scale bars are all $2 \mathrm{~nm}$.

the oxidation state of Ti by electron energy loss spectroscopy (EELS). Here we note that, although $O-K$ spectrum can directly provide information of oxygen vacancies, the much lower sensitivity of oxygen to the electron probe makes it difficult for detecting. Nevertheless, oxygen vacancies can be traced via their footprints in oxides including local lattice strain and electron doping (usually accompany with a valence reduction of Ti from $4+$ to $3+)^{19,39}$. Due to a coherently strained state of the heterostructure, the in-plane lattice strain (calculated by $\left(d_{[100]}-\bar{d}_{[100]}\right) / \bar{d}_{[100]}$, where $d_{[100]}$ is the lattice constant of each BTO unit cell measured from the STEM image and $\bar{d}_{[100]}$ is the corresponding lattice constant of bulk BTO) varies slightly throughout the BTO film (Supplementary Fig. 7). By contrast, the out-of-plane strain of the same region in Fig. 4d exhibits significant inhomogeneity (Fig. 4f). Considering the direct connection between oxygen vacancies and lattice expansion, the inhomogeneous out-of-plane lattice constant distribution thus reflects the clustering state of oxygen vacancies. Further comparing Fig. $4 \mathrm{~d}, \mathrm{f}$, we notice that oxygen vacancies tend to cluster at the vicinity of vortices. This phenomenon should be contributed by complex local fields accompanying oxygen vacancies. First of all, density functional theory calculations suggested that the local polarization vector neighboring a single oxygen vacancy can be reformed to point outward from the oxygen vacancy and away from the inherent polar axis due to lattice relaxation ${ }^{22,23,43}$. Second, the inhomogeneous strain coupled with oxygen vacancy clusters naturally gives rise to strain gradients and accompany flexoelectric fields, which also impact the arrangement of electric dipoles ${ }^{32,33}$. Lastly, a radial electrostatic field can be generated depending on the 
geometry of local charge arrangement, e.g., clustered ionized oxygen vacancies, which can contribute to the non-uniaxial polarization pattern as well ${ }^{13}$.

It is particularly worth mentioning that charged domain walls in the center of our BTO film are observed as well, which can also be induced by oxygen vacancies as reported previously ${ }^{44}$. The different patterns of electric dipoles induced by oxygen vacancies may depend on the specific geometries of oxygen vacancy clusters. For example, oxygen vacancy plates may give rise to charged domain walls ${ }^{44}$ while spherical oxygen vacancy clusters can induce swirling electric dipoles (as Domain I in Fig. 4d). Another interesting finding is that the region where oxygen vacancies populate show diminishing polarization. This contrasts the general belief of strong polarization-tetragonality coupling, but in consistence with previous calculations that predict the polarization suppressing role of oxygen vacancies ${ }^{24}$. Therefore, this finding calls for particular attention to the relation between lattice strain and local polarization values under the existence of oxygen vacancies.

Figure $4 \mathrm{~g}$ plots the Ti- $L_{2,3}$ spectra of two representative BTO regions with different polarization states, namely region-ofinterest (ROI)-1, -2 , and -3 in Fig. $4 \mathrm{~d}$. While all spectra show the characteristic $t_{2 g}-e_{g}$ splitting peaks of $L_{2}$ and $L_{3}$ edges for $\mathrm{Ti}^{4+}$, an additional peak emerges between $t_{2 g}$ and $e_{g}$ in the spectrum of ROI- 1 with large polarizations and has been ascribed to the $\mathrm{Ti}^{3+}$ state ${ }^{39}$. This result indicates admixture of $\mathrm{Ti}^{3+}$ and $\mathrm{Ti}^{4+}$ in ROI-1. It is worth noting that this feature is prevailing throughout the film, namely regions with large polarizations, e.g., the periphery of polar vortices, exhibit a reduced $\mathrm{Ti}$ valence state; regions with diminishing polarizations, e.g., the core of polar vortices (ROI-3) and charged domain walls (ROI-2), exhibit pure $\mathrm{Ti}^{4+}$. The dissociation of the oxygen vacancy clusters (from the local strain analysis) and the $\mathrm{Ti}^{3+}$-accumulated regions suggests certain delocalization of electrons doped by oxygen vacancies, which could be driven by polarization screening ${ }^{18,24}$. This finding again verifies the existence of oxygen vacancies and further suggests considerable inhomogeneity due to oxygen vacancy clusters in 5BTO films.

\section{DISCUSSION}

In the end, it is worth discussing the connection between the microscopic polarization patterns observed by STEM and those by PFM. The length scales of the polarization configurations by two methods may seem inconsistent, namely a typical scale of several nanometers in STEM and a scale of several to tens of nanometers in PFM. However, we note that PFM usually probes collective piezoelectric response over $100 \mathrm{~nm}$ deep into the target sample and evaluates the average local polarization under the tip $^{45}$. Therefore, in the case of complex polarization structures, e.g., $180^{\circ}$ domains with tail-to-tail domain walls and vortex-like polarization structures, PFM can only extract the sum of polarization from a large probing volume. Despite this drawback, we may still infer the existence of a complex polarization configuration in the 5-BTO film from PFM. For example, in Fig. 1d, both positively and negatively poled regions exhibited comparable PFM magnitudes whereas the pristine region with the same PFM phase as that of the positively poled region shows a much weaker magnitude. The weaker PFM magnitude can be interpreted as canceling of polarizations with opposite directions in an ensemble of complex polarization configurations such as rotating, head-tohead, or tail-to-tail polarization patterns, as observed by STEM $^{46}$. Nevertheless, by STEM, we indeed observe some regions with a mainly upward polarization (Supplementary Fig. 6a). Moreover, by analyzing the average polarizations of each unit-cell column in Fig. 4d, we can also observe a region with an average downward polarization and a lateral dimension of $\sim 10 \mathrm{~nm}$ (lower panel in Fig. $4 \mathrm{~d}$ and Supplementary Fig. 8). Therefore, our STEM and PFM results are mutually consistent. In this regard, STEM should provide a better knowledge of the polar structure, regardless of its limited observation area.

In summary, we report a close examination on the role of oxygen vacancies in the creation of swirling electric dipoles in a prototypical ferroelectric, namely $\mathrm{BaTiO}_{3}$, ultrathin film, which have been usually reported with a uniaxial polarization pattern due to the epitaxial strain. There are two important findings that have been overlooked in previous experimental studies. First, the dynamic clustering process of oxygen vacancies results in an evolution of a seemingly single domain to complex nanoscale polarization structures, as observed by PFM. This result calls for particular attention in understanding the self-polarization behavior of ferroelectric thin films with the presence of oxygen vacancies. Second, clustering oxygen vacancies can lead to nonuniaxial polarization structures such as vortices, which can be taken as embryo of topological polar objects. Notably, it was also reported that oxygen vacancies cluster at the core of vortex in the well-studied $\mathrm{PbTiO}_{3} / \mathrm{SrTiO}_{3}$ superlattice ${ }^{26}$. However, the formation of vortices in the $\mathrm{PbTiO}_{3} / \mathrm{SrTiO}_{3}$ superlattice has been regarded as a result of competition between the strain and the electrostatic boundary condition, and the positive role of oxygen vacancies has not been identified. Our study reveals a more active role of oxygen vacancies in producing rotating polarization structures. Moreover, the mobility of oxygen vacancies under external mechanical and electrical stimuli implies promising controllability over the coupled polarization structures ${ }^{47}$. From the aspect of practical applications, the electron-donor role of oxygen vacancies combined with the coupled topological polarization patterns should exert profound influences on the transport of ferroelectric oxide-based electronic devices, especially thin ferroelectric tunnel junctions and domain wall memresistors ${ }^{19,40}$. Therefore, our study should inspire future efforts on the exploration of topological polar objects by exploiting point defects.

\section{METHODS}

\section{Materials preparation and characterization}

Details of the PLD growth have been reported elsewhere ${ }^{29}$. AFM and PFM were performed using a Cypher scanning probe microscope (MFP-3D, Asylum Research) with Ir-Pt-coated tips (PPP-EFM, Nanosensors). A Dual Alternating Current Resonance Tracing mode was used for PFM measurements. XRD was performed with a Bruker D8 Discover. XPS was carried out in a VG ESCALAB 220i-XL system using a monochromatic Al Ka source with the pass energy of $10 \mathrm{eV}$.

\section{STEM characterization}

Cross-sectional STEM specimens were prepared by focused ion-beam milling (Helios 650, FEl) with low-energy ion beams $(<2 \mathrm{kV})$ at the final milling process and further thinned by focused low-energy Ar-ion milling (NanoMill 1040; Fischione Instruments). HAADF-STEM images for measuring atomic positions in this work were obtained by spherical aberration corrected STEM with a probe corrector (JEM-ARM 200F; JEOL Ltd) equipped with a cold field emission gun at an acceleration voltage of $200 \mathrm{kV}$ installed at the National Center for Inter-university Research Facilities at Seoul National University (South Korea). The semiconvergence angle for STEM analyses was set to $24 \mathrm{mrad}$. The inner semi-collection angle range was set to 80 and 30 mrad for HAADF- and LAADF-STEM, respectively. For suppressing spatial incoherency and improving signal-to-noise ratio of HAADF-STEM images, a series of images was acquired, with a fast scan speed of $1 \mu \mathrm{s}$ pixel $^{-1}$ for 30 frames, and registered to the atomic-resolved STEM images. Both rigid and non-rigid registration methods were applied and showed consistent results. The atomic positions were determined by fitting and subtracting them in a regular sequence of atomic number as 2D-Gaussian function using customized MATLAB code. Then polarizations of each BTO unit-cell were determined by using the column offsets of each titanium from the center of Ba cage. For all images, the horizontal and vertical lines represent the [100] and [001] orientations, respectively. EELS analyses were carried out 
at the corresponding locations to investigate the relationship between polarization domain structure and electronic structure using an EEL spectrometer (Model 965 GIF Quantum ER, Gatan, USA) with an energy resolution of $0.8 \mathrm{eV}$.

\section{DATA AVAILABILITY}

The data and code that support the findings of this study are available from the corresponding author upon reasonable request.

Received: 2 February 2021; Accepted: 19 April 2021; Published online: 13 May 2021

\section{REFERENCES}

1. Vasudevan, R. K. et al. Exploring topological defects in epitaxial $\mathrm{BiFeO}_{3}$ thin films. ACS Nano 5, 879-887 (2011).

2. Nelson, C. T. et al. Spontaneous vortex nanodomain arrays at ferroelectric heterointerfaces. Nano Lett. 11, 828-834 (2011).

3. Nahas, Y. et al. Discovery of stable skyrmionic state in ferroelectric nanocomposites. Nat. Commun. 6, 8542 (2015).

4. Tang, Y. L. et al. Observation of a periodic array of flux-closure quadrants in strained ferroelectric $\mathrm{PbTiO}_{3}$ films. Science 348, 547-551 (2015).

5. Yadav, A. K. et al. Observation of polar vortices in oxide superlattices. Nature $\mathbf{5 3 0}$ 198-201 (2016).

6. Zhang, Q. et al. Nanoscale bubble domains and topological transitions in ultrathin ferroelectric films. Adv. Mater. 29, 1702375 (2017).

7. $\mathrm{Li}, \mathrm{Z}$. et al. High-density array of ferroelectric nanodots with robust and reversibly switchable topological domain states. Sci. Adv. 3, e1700919 (2017).

8. Geng, W. et al. Rhombohedral-orthorhombic ferroelectric morphotropic phase boundary associated with a polar vortex in $\mathrm{BiFeO}_{3}$ films. ACS Nano 12 11098-11105 (2018).

9. Pereira Gonçalves, M. A., Escorihuela-Sayalero, C., Garca-Fernández, P., Junquera, J. \& Íñiguez, J. Theoretical guidelines to create and tune electric skyrmion bubbles. Sci. Adv. 5, eaau7023 (2019).

10. Das, S. et al. Observation of room-temperature polar skyrmions. Nature 568, 368-372 (2019)

11. Wang, Y.-J., Zhu, Y.-L. \& Ma, X.-L. Chiral phase transition at $180^{\circ}$ domain walls in ferroelectric $\mathrm{PbTiO}_{3}$ driven by epitaxial compressive strains. J. Appl. Phys. 122, 134104 (2017)

12. Wang, Y. J. et al. Polar meron lattice in strained oxide ferroelectrics. Nat. Mater 19, 881-886 (2020).

13. Li, L. et al. Defect-induced hedgehog polarization states in multiferroics. Phys. Rev Lett. 120, 137602 (2018).

14. Pramanick, A., Prewitt, A. D., Forrester, J. S. \& Jones, J. L. Domains, domain walls and defects in perovskite ferroelectric oxides: a review of present understanding and recent contributions. Critic Rev. Solid State Mater. Sci. 37, 243-275 (2012).

15. Herklotz, A. et al. Strain coupling of oxygen non-stoichiometry in perovskite thin films. J. Phys. Condens. Matter 29, 493001 (2017).

16. Tagantsev, A. K., Stolichnov, I., Colla, E. L. \& Setter, N. Polarization fatigue in ferroelectric films: basic experimental findings, phenomenological scenarios, and microscopic features. J. Appl. Phys. 90, 1387-1402 (2001).

17. Jia, C. L. \& Urban, K. Atomic-resolution measurement of oxygen concentration in oxide materials. Science 303, 2001-2004 (2004).

18. Lee, D. et al. Active control of ferroelectric switching using defect-dipole engineering. Adv. Mater. 24, 6490-6495 (2012).

19. Sanchez-Santolino, G. et al. Resonant electron tunneling assisted by charged domain walls in multiferroic tunnel junctions. Nat. Nanotechnol. 12, 655-662 (2017).

20. Ren, X. Large electric-field-induced strain in ferroelectric crystals by point-defectmediated reversible domain switching. Nat. Mater. 3, 91-94 (2004).

21. Kimmel, A. V., Weaver, P. M., Cain, M. G. \& Sushko, P. V. Defect-mediated lattice relaxation and domain stability in ferroelectric oxides. Phys. Rev. Lett. 109, 117601 (2012).

22. Shimada, T., Ueda, T., Wang, J. \& Kitamura, T. Hybrid Hartree-Fock density func tional study of charged point defects in ferroelectric $\mathrm{PbTiO}_{3}$. Phys. Rev. B 87 174111 (2013).

23. Li, M., Li, J., Chen, L.-Q., Gu, B.-L. \& Duan, W. Effects of strain and oxygen vacancies on the ferroelectric and antiferrodistortive distortions in $\mathrm{PbTiO}_{3} / \mathrm{SrTiO}_{3}$ superlattice. Phys. Rev. B 92, 115435 (2015).

24. Akbarian, D. et al. Understanding the influence of defects and surface chemistry on ferroelectric switching: a ReaxFF investigation of $\mathrm{BaTiO}_{3}$. Phys. Chem. Chem Phys. 21, 18240-18249 (2019)
25. Kim, Y.-M. et al. Direct observation of ferroelectric field effect and vacancycontrolled screening at the $\mathrm{BiFeO}_{3} / \mathrm{LaxSr}_{1-x} \mathrm{MnO}_{3}$ interface. Nat. Mater. 13 1019-1025 (2014).

26. Du, K. et al. Manipulating topological transformations of polar structures through real-time observation of the dynamic polarization evolution. Nat. Commun. 10, 4864 (2019).

27. Fong, D. D. et al. Stabilization of monodomain polarization in ultrathin $\mathrm{PbTiO}_{3}$ films. Phys. Rev. Lett. 96, 127601 (2006).

28. Chen, J. et al. Upward ferroelectric self-polarization induced by compressive epitaxial strain in (001) $\mathrm{BaTiO}_{3}$ films. J. Appl. Phys. 113, 204105 (2013).

29. Peng, W. et al. Constructing polymorphic nanodomains in $\mathrm{BaTiO}_{3}$ films via epitaxial symmetry engineering. Adv. Funct. Mater. 30, 1910569 (2020).

30. Lu, H. et al. Mechanical writing of ferroelectric polarization. Science 336, 59-61 (2012).

31. Lee, $\mathrm{H}$. et al. Imprint control of $\mathrm{BaTiO}_{3}$ thin films via chemically induced surface polarization pinning. Nano Lett. 16, 2400-2406 (2016).

32. Lee, D. et al. Giant flexoelectric effect in ferroelectric epitaxial thin films. Phys. Rev. Lett. 107, 057602 (2011).

33. Guo, R. et al. Tailoring self-polarization of $\mathrm{BaTiO}_{3}$ thin films by interface engineering and flexoelectric effect. Adv. Mater. Interf. 3, 1600737 (2016).

34. Jeon, B. C. et al. Flexoelectric effect in the reversal of self-polarization and associated changes in the electronic functional properties of $\mathrm{BiFeO}_{3}$ thin films. Adv. Mater. 25, 5643-5649 (2013).

35. Yu, P. et al. Interface control of bulk ferroelectric polarization. Proc. Natl Acad. Sci. U. S. A. 109, 9710-9715 (2012).

36. Kholkin, A. L., Brooks, K. G., Taylor, D. V., Hiboux, S. \& Setter, N. Self-polarization effect in $\mathrm{Pb}(\mathrm{Zr}, \mathrm{Ti}) \mathrm{O}_{3}$ thin films. Integr. Ferroelectr. 22, 525-533 (1998).

37. Mtebwa, M., Feigl, L. \& Setter, N. Joint IEEE International Symposium on Applications of Ferroelectric and Workshop on Piezoresponse Force Microscopy (ISAF/ PFM) 73-75 (2013).

38. Eom, K. et al. Oxygen vacancy linear clustering in a perovskite oxide. J. Phys. Chem. Lett. 8, 3500-3505 (2017).

39. Muller, D. A., Nakagawa, N., Ohtomo, A., Grazul, J. L. \& Hwang, H. Y. Atomic-scale imaging of nanoengineered oxygen vacancy profiles in $\mathrm{SrTiO}_{3}$. Nature 430 657-661 (2004)

40. Hashimoto, S., Sugie, T., Zhang, Z., Yamashita, K. \& Noda, M. Effects of final annealing in oxygen on characteristics of $\mathrm{BaTiO}_{3}$ thin films for resistance random access memory. Jpn. J. Appl. Phys. 54, 10NA12 (2015).

41. Sheng, J., Fukami, T. \& Karasawa, J. X-ray photoemission spectroscopy (XPS) and thermally stimulated current (TSC) studies on the resistance degradation of irondoped titania ceramics. J. Am. Ceram. Soc. 81, 260-262 (1998).

42. Kwei, G. H., Lawson, A. C., Billinge, S. J. L. \& Cheong, S. W. Structures of the ferroelectric phases of barium titanate. J. Phys. Chem. 97, 2368-2377 (1993)

43. Wang, J. et al. Effects of oxygen vacancies on polarization stability of barium titanate. Sci. China Phys. Mech. 59, 634602 (2016).

44. Geng, W. R. et al. Unveiling the pinning behavior of charged domain walls in $\mathrm{BiFeO}_{3}$ thin films via vacancy defects. Acta Mater. 186, 68-76 (2020).

45. Soergel, E. Piezoresponse force microscopy. J. Phys. D. Appl. Phys. 44, 464003 (2011).

46. Kim, T. H. et al. Continuous control of charge transport in $\mathrm{Bi}$-deficient $\mathrm{BiFeO}_{3}$ films through local ferroelectric switching. Adv. Funct. Mater. 22, 4962-4968 (2012).

47. Das, S. et al. Controlled manipulation of oxygen vacancies using nanoscale flexoelectricity. Nat. Commun. 8, 615 (2017).

\section{ACKNOWLEDGEMENTS}

This work was supported by the Research Center Program of IBS (Institute for Basic Science) in Korea (IBS-R009-D1). L. W. acknowledges the support by the National Key Basic Research Program of China (Grant Nos. 2020YFA0309100) and the National Natural Science Foundation of China (Grant Nos. 12074365). STEM measurement was supported by the National Center for Inter-University Research Facilities (NCIRF) at Seoul National University in Korea and the National Research Foundation of Korea (NRF) grant funded by the Korea government (MSIT, No. 2017R1A2B3011629 and No. 2020R1A5A6017701). Q. X. and J. C. acknowledge the support from the Singapore Minister of Education (Grant No. Tier 1R284000195114) and A*STAR (Grant No. A1983c0036).

\section{AUTHOR CONTRIBUTIONS}

W.P. conceived the idea and designed the experiments. T.W.N. directed the whole research. W.P. and L.W. grew the films and performed PFM, XRD, and AFM measurements. J.M. and M.K. performed STEM measurements. Q.X. and J.C performed XPS measurements. W.P. and T.W.N. wrote the paper. All the authors contributed to the discussion and manuscript preparation. 


\section{COMPETING INTERESTS}

The authors declare no competing interests.

\section{ADDITIONAL INFORMATION}

Supplementary information The online version contains supplementary material available at https://doi.org/10.1038/s41535-021-00349-y.

Correspondence and requests for materials should be addressed to W.P. or T.W.N.

Reprints and permission information is available at http://www.nature.com/reprints

Publisher's note Springer Nature remains neutral with regard to jurisdictional claims in published maps and institutional affiliations.
Open Access This article is licensed under a Creative Commons Attribution 4.0 International License, which permits use, sharing, adaptation, distribution and reproduction in any medium or format, as long as you give appropriate credit to the original author(s) and the source, provide a link to the Creative Commons license, and indicate if changes were made. The images or other third party material in this article are included in the article's Creative Commons license, unless indicated otherwise in a credit line to the material. If material is not included in the article's Creative Commons license and your intended use is not permitted by statutory regulation or exceeds the permitted use, you will need to obtain permission directly from the copyright holder. To view a copy of this license, visit http://creativecommons. org/licenses/by/4.0/.

(c) The Author(s) 2021 